ARTICLE

\title{
Diagnosis of food allergy: History, examination and in vivo and in vitro tests
}

\author{
D A van der Spuy, A J Terblanche, S Karabus, M Kriel, A I Manjra, E Goddard, C L Gray, A C Lang, S M Risenga, M E Levin, \\ for the South African Food Allergy Working Group (SAFAWG)
}

All authors' degrees, affiliations and conflict of interest statements can be found online at http://dx.doi.org/10.7197/SAMJ.9201

Corresponding author: M Levin (michael.levin@uct.ac.za)

One cannot depend on one single test to diagnose food allergy. A detailed history is an essential initial step in cases of suspected food allergy. Aspects of the history should be gathered separately for each food being considered, as a patient may experience different types of reactions with various foods, each of which requires individual diagnostic and management strategies. History alone is not diagnostic and additional measures of sensitisation or food challenges are often required.

In suspected immunoglobulin E (IgE)-mediated allergy, skin-prick tests (SPTs) and/or measurement of serum specific IgE antibodies (ImmunoCAP) to suspected foods is used to prove sensitisation. Sensitisation does not, however, confirm clinical food allergy as these tests indicate an immunological response to the specific allergen, but the diagnosis requires a clear correlation between the test result and clinical reaction (by positive history or food challenge). The magnitude of the test result (SPT mean wheal size or ImmunoCAP level in $\mathrm{kU} / \mathrm{L}$ ) correlates with the likelihood of clinical allergy, but not the severity of a reaction.

Choice of the allergens tested should be guided by the history, but limited to the lowest necessary number to avoid false-positive results. Tests for sensitisation to foods should not be performed when the history indicates that such foods are tolerated. Ninety-five per cent positive predictive values (where a clinical reaction can be predicted in $95 \%$ of cases) have been described for immediate reactions, but may be population specific.

There are no validated tests to confirm non-IgE- or mixed IgE- and non-IgE-mediated food allergies. Diagnosis of this group of allergies depends on elimination of the suspected food, clearance of symptoms, and recurrence of symptoms on re-introduction of the food.

S Afr Med J 2015;105(1):69-70. DOI:10.7196/SAMJ.9100

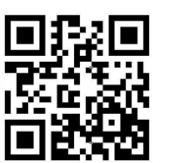

\section{History}

A detailed history is an essential initial step when a food allergy is suspected. ${ }^{[1]}$ This will help to determine the likelihood of and type of food-induced allergy and potential causative food/s. History alone is not diagnostic. In combination with the results of the specific immunoglobulin E (IgE) tests or skin-prick tests (SPTs), the likelihood of clinical food allergy can be identified. ${ }^{[2]}$ Aspects of the history should be gathered separately for each food being considered, as a patient may experience different types of reactions with various foods, each of which will require individual diagnostic and management strategies.

Information gathered during the history should include an allergy-focused family history, ${ }^{[2-4]}$ clinical history, ${ }^{[3,5,6]}$ and dietary history. ${ }^{[3]}$ Table 1 outlines the specific information required from the history.

\section{Examination}

The physical examination focuses on the signs of acute allergy, should the patient be examined during an acute reaction, or the stigmata of chronic allergic disease when the patient presents with chronic symptoms. Acute signs may include urticaria and angio-oedema, wheeze, cough, rhinorrhoea, diarrhoea, vomiting, and cardiovascular signs such as hypotension and tachycardia.

A general examination may reveal allergic facies, allergic salute and pallor. Nutritional status should be assessed by plotting height, weight and weight-for-height. Signs of nutritional deficiencies, such as iron deficiency or rickets, should be sought.

The skin is examined for signs of atopic eczema and urticaria. Mouth breathing, allergic mannerisms, nasal congestion, dental abnormalities and post-nasal drip are looked for in the upper airways, while the chest is examined for hyperinflation and wheeze.

\section{Diagnostic tests}

One cannot depend on one single test to diagnose food allergy. ${ }^{[5]}$ A history aids in identifying potentially involved food when differentiating between potential IgE- and non-IgE-mediated disease and assessing the severity of reactions. In suspected IgE-mediated allergy, SPTs and/or measurement of serum specific IgE antibodies (ImmunoCAP) to suspected foods is used to prove sensitisation. Sensitisation does not, however, confirm clinical food allergy, as these tests indicate an immunological response to the specific allergen, but the diagnosis requires a clear correlation between the test result and clinical reaction (by positive history or food challenge). ${ }^{[12]}$ Blind testing without suspicion of a reaction to a specific allergen is not advocated - findings are often misleading as they indicate asymptomatic sensitisation alone. ${ }^{[13]}$ Tests for sensitisation to foods should not be performed when history indicates that those foods are tolerated.

There are no validated tests to confirm non-IgE- or mixed IgEand non-IgE-mediated food allergies. Diagnosis of this group of allergies depends on elimination of the suspected food, clearance of symptoms, and a re-introduction of the food as an oral food challenge (OFC). In certain cases endoscopy with biopsy is indicated to evaluate the response to dietary changes.

\section{Diagnostic tests to identify sensitisation in IgE-mediated food allergy \\ Skin-prick tests}

An SPT is a useful and scientifically valid tool to confirm IgEmediated sensitisation to a specific allergen. Skin-testing directly 
Table 1. Information gathering in history-taking

Family history
Clinical history
Personal history
Age
Occupation- and
environmental-associated risks
Symptom type

Presence of first-degree relatives with any atopic disease (asthma, eczema or allergic rhinitis) or food allergy

History of atopic disease

Relationship of person who raised the concern of food allergy

Age at first exposure to the food, any subsequent exposures and timing and type of all reactions

Possible exposures at work/crèche/school/home

Symptoms associated with IgE-mediated reactions (may differ according to severity of reaction)

- Urticaria, angio-oedema

- Oral itching

- Abdominal pain, nausea, vomiting

- Nasal symptoms: congestion, sneezing, rhinorrhoea

- Respiratory symptoms, e.g. difficulty breathing, wheezing, persistent coughing, voice change

- Floppiness, change in level of consciousness, anxiety, sleepiness

- Cyanosis, loss of consciousness, hypotension

Symptoms associated with non-IgE-mediated reactions (may differ according to type and severity of reaction)

- Lower gastrointestinal symptoms: diarrhoea, failure to thrive, malabsorption, protein-losing enteropathy, abdominal pain, bloody stools, constipation, cramps or colic

- Gastro-oesophageal reflux disease, dysphagia, odynophagia, vomiting

- Atopic dermatitis not responding to maximal appropriate topical therapy

- Pallor, signs of macro- and micronutrient deficiencies

Timing of reaction

Timing of symptoms after exposure to the food, which could indicate possible immunological reaction

- Immediate: minutes up to 2 hours (usually IgE mediated)

- Delayed or late onset: $>2$ hours (non-IgE mediated)

- Both immediate and delayed reactions

Reaction details

Duration

Severity

- Mild to life-threatening

- Reaction severity compared with previous reaction

Frequency and whether the reactions have occurred previously

Causative food allergen

Dose of allergen

Form of food

Setting

Reproducibility

Route of allergen exposure

Concomitant diseases

Co-factors

Co- and cross-reactivity

Treatment

\section{Dietary history}

Growth and development

Suspected food/s causing the reaction and simultaneous ingestion of other food/s or ingredients

Level of food allergen exposure causing a reaction

Processed/canned and/or raw/cooked/heat-treated/dried food

Details where the reaction occurred

Reaction repeatability and consistency each time the offending food is given

Route of exposure

- Oral, via breastmilk or other food/feed

- Inhalation

- Skin contact

Presence of other medical conditions, including asthma or atopic diseases

Exercise or ingestion of alcohol, coffee or drugs before or after eating the offending food

Tolerance of or reaction to related food allergens

Cross-reactivity between inhalant allergens and food allergens ${ }^{[5,7]}$

Common examples

- Peanuts, with cross-reactive allergy to one or more tree nuts ${ }^{[8]}$ and/or sesame, ${ }^{[10]}$ or co-reactivity between peanut and egg ${ }^{[9]}$

- Pollen-food syndrome, with reactions to many different fruits and vegetables ${ }^{[7]}$

- Latex allergens can cross-react with kiwi, avocado, banana and others ${ }^{[1]}$

Details on how the symptoms were treated, medication used and time to symptom resolution

Check growth records of the infant/child

Identify cause of poor growth, whether related to food allergy or other cause 
Table 1. (continued) Information gathering in history-taking

\begin{tabular}{|c|c|}
\hline $\begin{array}{l}\text { Referral to other healthcare } \\
\text { providers }\end{array}$ & $\begin{array}{l}\text { Identify the need for referral to other healthcare professionals for assessment, e.g. a dietician to ensure } \\
\text { nutritional adequacy; speech and language therapist and/or occupational therapist with expertise in sensory or } \\
\text { behavioural issues related to poor food intake }\end{array}$ \\
\hline Feeding history & $\begin{array}{l}\text { Feeding history, duration of breastfeeding, type of infant formula given, age of solid food introduction, food } \\
\text { aversion/refusal, which may indicate food allergy related to pain or discomfort on exposure }\end{array}$ \\
\hline Dietary intake & $\begin{array}{l}\text { Dietary intake should be assessed with a detailed dietary history, a 3-day food record, a } 24 \text {-hour recall or a } \\
\text { typical day's intake }\end{array}$ \\
\hline Cultural and religious factors & Assess cultural and religious factors that affect the patient's diet \\
\hline $\begin{array}{l}\text { Incidental or accidental } \\
\text { ingestion }\end{array}$ & Ensure awareness of hidden sources of the offending food \\
\hline Other foods & $\begin{array}{l}\text { Ability of the patient to eat a full age-appropriate portion of ... (name specific commonly allergenic foods as } \\
\text { well as cross-reactive foods) }\end{array}$ \\
\hline Elimination diets & $\begin{array}{l}\text { Foods avoided and reasons for avoidance } \\
\text { Results of previous elimination diets in alleviating symptoms and/or identifying the offending food }\end{array}$ \\
\hline Supplements & Dosage, type and manufacturer of supplements taken to cover for missing nutrients \\
\hline
\end{tabular}

Table 2. Food allergy skin prick test decision points ${ }^{[14]}$

\begin{tabular}{lll}
\hline Food allergen & $\begin{array}{l}\mathbf{1 0 0 \%} \mathbf{P P V}<\mathbf{2} \text { y } \\
\text { (wheal diameter) }\end{array}$ & $\begin{array}{l}\mathbf{1 0 0 \%} \text { PPV }>\mathbf{2} \text { y } \\
\text { (wheal diameter) }\end{array}$ \\
\hline Cow's milk & $6 \mathrm{~mm}$ & $>8 \mathrm{~mm}$ \\
Egg & $5 \mathrm{~mm}$ & $>7 \mathrm{~mm}$ \\
Peanut & $4 \mathrm{~mm}$ & $>8 \mathrm{~mm}$ \\
PPV $=$ positive predictive value. & &
\end{tabular}

assesses the presence of mast-cell-bound allergen-specific IgE. Allergen extracts, prepared commercially or directly from the suspected food, are introduced into the skin. A reactive SPT is a sign of sensitisation only, and cannot be considered diagnostic without clear clinical correlation and/or confirmation with an OFC. This test is simple, with rapid results, good performance, low cost and high sensitivity. ${ }^{[12]}$ The allergens tested for should be guided by the history, but also need to be limited to the lowest necessary number to avoid false-positive results. Antihistamines should be discontinued 3 - 5 days before the test. SPTs should be performed in a setting where equipment and medication are available to treat rare, severe reactions, including anaphylaxis.

The mean wheal diameter correlates with the likelihood of clinical allergy, but must be interpreted in light of the clinical history. In itself it is not diagnostic and cannot predict the severity of reactions. Individuals with a clear history of a severe reaction to a particular food should not undergo skin-testing to that food to prove sensitisation, because where there is uncertainty and an increased risk of a severe reaction, such risks may exceed the benefit. In individuals with atopic dermatitis, positive predictive values (cut-off values for the mean wheal diameter in the common food allergens where a clinical reaction can be predicted in the specified proportion of cases) have been described for immediate reactions (Table 2), but may be population specific.

\section{Allergen-specific serum IgE}

Serum IgE specific to a particular allergen can be determined by immunoassays (ImmunoCAP). These assays are validated, reliable and reproducible, but costly. Cut-off values for the $95 \%$ positive predictive values of a clinical reaction to common food allergens are given in Table 3, but may be population specific. ${ }^{[13]}$
Table 3. Food allergy ImmunoCAP decision points

\begin{tabular}{|c|c|c|}
\hline Food allergen & Decision point (kUA/L) & PPV, \% \\
\hline \multicolumn{3}{|l|}{ Cow's milk } \\
\hline$<1 y^{[15]}$ & 5 & 95 \\
\hline$>2 y^{[16]}$ & 15 & 95 \\
\hline \multicolumn{3}{|l|}{ Egg } \\
\hline$<2 y^{[17]}$ & 2 & 95 \\
\hline$>2 y^{[16]}$ & 7 & 98 \\
\hline Peanut ${ }^{[16]}$ & 14 & 100 \\
\hline Fish $^{[16]}$ & 20 & 100 \\
\hline Wheat ${ }^{[16]}$ & 26 & 74 \\
\hline Soya ${ }^{[16]}$ & 30 & 73 \\
\hline Tree nuts $^{[16]}$ & 15 & 95 \\
\hline $\mathrm{PPV}=$ positive pred & & \\
\hline
\end{tabular}

Purified recombinant allergen-specific IgE tests against individual major allergen components in food may improve the diagnosis of clinical allergy and differentiate true food allergy from cross-reactivity. Certain component allergens are correlated with persistence of food allergy (e.g. ovomucoid in egg and casein in cow's milk protein allergy), severity of reactions (e.g. Ara h 2 in peanut allergy ${ }^{[5]}$ and whether heat-degraded protein will be tolerated (e.g ovomucoid and casein).

The assessment of co- and cross-sensitisation to related food or aeroallergens should be considered. Cross-reactive food allergies such as pollen food syndrome and latex food allergy are common. Only food and aeroallergens related to the specific patient's presentation should be tested to prevent mislabelling of asymptomatic sensitisation alone as clinically relevant food allergy.

\section{Total serum IgE}

Total serum IgE is not recommended in the diagnosis of food allergy and should not be done. ${ }^{[3]}$

\section{Atopy patch test}

The atopy patch test has been developed to aid diagnosis in delayed reactions in patients with atopic dermatitis and eosinophilic 
oesophagitis. This test has, however, not been validated or standardised and is not recommended for the routine diagnosis of food allergy. ${ }^{[2]}$

\section{Intradermal tests}

Intradermal skin tests have a low specificity and high risk for systemic and irritant reactions. They are not recommended for food allergy testing. ${ }^{[2,3]}$

\section{Non-standardised and unproven procedures}

Unconventional tests for allergy diagnosis used by complementary and alternative health professionals include IgG and IgG4 testing, kinesiology or applied kinesiology, iridology, cytotoxic (antigen leukocyte cellular antibody test (ALCAT)/Bryan's) testing, Vega (electrodermal) testing, and hair analysis. These tests are unvalidated, poorly reproducible, have poor predictive value for allergies and cannot diagnose sensitisation. Practices that claim to affect a cure by removing the patient's blood, processing it and then re-infusing it into the patient (autohaemotherapy) are strongly discouraged.

Such tests may be harmful to patients by leading to under- or overdiagnosis of food allergy. False-negative results from unconventional tests may prompt patients with clinically important food allergy to continue to ingest foods that are harmful to them, and conversely false-positive results may prompt multiple dietary exclusions and cause significant nutritional impairment. ${ }^{[18]}$ In addition, such tests are often extremely expensive.

References

1. Koletzko S, Niggemann B, Arato A, et al. Diagnostic approach and management of cow's-milk protein allergy in infants and $\mathrm{B}$ ildren $\mathrm{A}$. $\mathrm{A}, \mathrm{e}$ ( Nutr 2012;55(2):221-229. [http://dx.doi.org/10.1097/01.mpg.0000181841.07090.f4]
2. European Academy of Allergy and Clinical Immunology (EAACI). EAACI Food Allergy and Anaphylaxis Guidelines: Diagnosis and management of food allergy. http://www.eaaci.org/resources/ food-allergy-and-anapyhlaxis-guidelines.html (accessed 17 November 2014)

3. Boyce JA, Assaad A, Burks AW, et al. Guidelines for the diagnosis and management of food allergy Boyce JA, Assaad A, Burks AW, et al. Guidelines for the diagnosis and management of food allergy
in the United States. J Allergy Clin Immunol 2010;126(suppl):S1-S58. [http://dx.doi.org/10.1016/j. jaci.2010.10.008]

4. Centre for Clinical Practice at NICE (UK). Food Allergy in Children and Young People: Diagnosis and Assessment of Food Allergy in Children and Young People in Primary Care and Community Settings. London: National Institute for Health and Clinical Excellence (UK), 2011

5. Burks AW, Tang M, Sicherer S, et al. ICON: Food allergy. J Allergy Clin Immunol 2012;129(4):906-920. [http://dx.doi.org/10.1016/j.jaci.2012.02.001]

6. Fiocchi A, Brozek J, Schünemann H, et al. World Allergy Organization (WAO) Diagnosis and Rationale for Action against Cow's Milk Allergy (DRACMA) Guidelines. World Allergy Organ J 2010;3(4):57-161. [http://dx.doi.org/10.1097/WOX.0b013e3181defeb9]

7. Kondo Y, Urisu A. Oral allergy syndrome. Allergol Int 2009;58(4):485-491. [http://dx.doi.org/10.2332/ allergolint.09-RAI-0136

8. De Leon MP, Glaspole IN, Drew AC, Rolland JM, O'Hehir RE, Suphioglu C. Immunological analysis of allergenic cross-reactivity between peanut and tree nuts. Clin Exp Allergy 2003;33(9):1273-1280. [http://dx.doi.org/10.1046/j.1365-2222.2003.01761.x]

9. Stutius LM, Sheehan WJ, Rangsithienchai P, et al. Characterizing the relationship between sesame, coconut and nut allergy in children. Pediatr Allergy Immunol 2010; 21(8):1114-1118. [http://dx.doi.
com org/10.1111/j.1399-3038.2010.00997.x]

10. Du Toit G, Santos A, Roberts G, Fox AT, Smith P, Lack G. The diagnosis of IgE-mediated food allergy in childhood. Pediatr Allergy Immunol 2009;20(4):309-319. [http://dx.doi.org/10.1111/ 1399-3038.2009.00887.x

11. Gawchik S. Latex allergy. Mt Sinai J Med 2011;78(5):759-772. [http://dx.doi.org/10.1002/msj.20281]

2. Eigenmann PA, Oh JW, Beyer K. Diagnostic testing in the evaluation of food allergy. Pediatr Clin N Am 2011;58:351-362. [http://dx.doi.org/10.1002/msj.20281]

13. Gupta RS, Dyer AA, Jain N, Greenhawt MJ. Childhood allergies: Current diagnosis, treatment, and management strategies. Mayo Clin Proc 2013;88(5):512-526. [http://dx.doi.org/10.1016/j. mayocp.2013.03.005

14. Sporik R, Hill DJ, Hosking CS. Specificity of allergen skin testing in predicting positive open food challenges to milk, egg and peanut in children. Clin Exp Allergy 2000;30(11):1540-1546. [http://dx.doi. org/10.1046/j.1365-2222.2000.00928.x]

15. Garcia-Ara C, Boyano-Martinez T, Diaz-Pena JM, et al. Specific IgE levels in the diagnosis of immediate hypersensitivity to cow's milk protein in the infant. J Allergy Clin Immunol 2001;107(1):185-190.

16. Sampson HA. Utility of food-specific IgE concentrations in predicting symptomatic food allergy. J Allergy Clin Immunol 2001;107(5):891-896. [http://dx.doi.org/10.1067/mai.2001.114708]

17. Boyano-Martinez T, Garcia-Ara C, Diaz-Pena JM, et al. Prediction of tolerance on the basis of quantification of egg white-specific IgE antibodies in children with egg allergy. J Allergy Clin Immunol 2002;110(2):304-309. [http://dx.doi.org/10.1067/mai.2002.126081]

18. Noimark L, Cox HE. Nutritional problems related to food allergy in childhood. Pediatr Allergy Immunol 2008;19(2):188-195. [http://dx.doi.org/10.1111/j.1399-3038.2007.00700.x] 